\title{
Statistical Properties of Magnetic CP Stars
}

\author{
S. Bagnulo \\ European Southern Observatory, Alonso de Cordova 3107, Santiago, \\ Chile
}

\begin{abstract}
Magnetic fields of chemically peculiar (CP) stars of the upper main sequence are characterised by a geometry organised at a large scale, permeating the entire photosphere, and with a typical strength of the order of $0.1-30 \mathrm{kG}$. Here I review the results obtained from statistical studies of the structures of the magnetic fields of CP stars, which are aimed at finding how magnetic strength and morphology are correlated with other stellar characteristics and with the star's evolutionary state.
\end{abstract}

\section{Introduction}

About $10-15 \%$ of upper main sequence stars are chemically peculiar, in the sense that the abundances of some elements are remarkably different to the solar case. Some rare-earth elements exhibit abundance values that may be up to tens, hundreds, or thousands of times higher than what observed for instance in the Sun. Iron-peak elements are also often characterised by peculiar abundances, although the difference with respect to the abundances of normal A and B stars is usually less than 1 dex. These stars are commonly referred to as Ap and Bp stars, or, according to the classification by Preston (1974), CP (chemically peculiar) stars.

It is commonly accepted that the measured overabundances (or, in some cases, underabundances) do not reflect a peculiar chemical composition of the entire star, but only of the stellar photosphere. In fact, there is strong evidence for non homogeneous element distribution even within the photosphere itself, not only horizontally (the presence of abundance patches has been known for a long time) but also vertically (Babel \& Lanz 1992, Bagnulo et al. 2001, Ryabchikova et al. 2002). The process that explains these abundance inhomogeneities is microscopic chemical diffusion: the distribution of the elements is the result of a competition between gravitational settling and radiative pressure guided by the presence of a magnetic field (Michaud 1970, Alecian \& Stift 2002).

A recently discovered peculiarity is that profiles of $\mathrm{H} \alpha$ in a number of cool $\mathrm{CP}$ stars show broad wings ending abruptly in narrow cores. Wings are consistent with temperatures in the range $7000-8000 \mathrm{~K}$, whereas core widths are consistent with temperatures of $6000 \mathrm{~K}$ or lower (Cowley et al. 2001). An empirical interpretation of this phenomenon in terms of a modified temperature distribution has been given by Kochukhov et al. (2002a), who speculated that the anomalous temperature distribution results from a strong stratification of metal lines. 
CP stars have generally longer rotation periods than normal A and B stars. Rotation periods of CP stars can be of a few days, weeks, or months. The bulk of their rotation rates forms a separate Maxwellian distribution with an average value 3-4 times lower than that found in normal $\mathrm{A}$ and B-type stars (Stępien 2000), but there also exist groups of CP stars with rotation period of years (Mathys et al. 1997) and even several decades (e.g., $\gamma$ Equ, see Leroy et al. 1994). Several works suggest that field Ap stars do not undergo any significant magnetic braking during their life on the Main Sequence (e.g., North 1998), which therefore must occur before the star reaches the Zero Age Main Sequence. Stȩpien (2000) explains the slow rotation as the result of an interaction of the stellar magnetic field with the circumstellar environment during the pre-main sequence phase. And if a magnetized wind still persists after the dissipation of the circumstellar disk, a pre-main sequence CP star may further slow down, reaching the Zero Age Main Sequence with an extremely long rotation period.

One of the most interesting phenomena observed in CP stars is that their spectra are polarized, and that the way spectral lines are polarized changes periodically with time. Magnetic field is the obvious agent responsible for the polarization of the spectral lines, and the reason why the polarization is periodically changing is that the magnetic morphology is not symmetric about the star's rotation axis, so that the observer sees a magnetic configuration that changes as the star rotates. This is the Oblique Rotator Model, originally proposed by Babcock (1949) and by Stibbs (1950). An important difference with respect to the case of late-type stars should be noted. Magnetic fields of late-type stars tend to be organized at a small scale, for instance in relatively small spots like in the case of the Sun, whereas the magnetic field of early-type stars is organized at a large scale, permeating the entire stellar photosphere, with a typical strength between a few hundred and a few tens of thousands of Gauss. It is believed that most of CP stars are magnetic, in particular most or probably all Ap SrCrEu, Ap Si, He-weak, He-strong, SiSrTi stars are magnetic, whereas the Ap HgMn, Am, He-weak PGa, and $\lambda$ Bootis stars are not magnetic at all, or, if they are, their fields are not ordered as those of the magnetic Ap stars (Shorlin et al., 2002). Finally, there is no convincing evidence for the existence of magnetic fields in early-type stars that are not chemically peculiar (Shorlin et al., 2002) (but see Hubrig \& Castelli 2001 for a different point of view).

One of the tasks to be undertaken in order to understand the physics of $\mathrm{CP}$ stars is the modelling of their magnetic structures. For instance, finding a relationship between magnetic field topology and geographic distribution of the elements would give constraints to the diffusion theory; finding relationships between magnetic field morphology and other stellar parameters would help to explain the problem of the origin of the magnetic field. The aim of this contribution is to review the current status of the results of the modelling of magnetic fields of early-type stars.

Polarimetric techniques are the basic tools for studying the morphologies of stellar magnetic fields, thus in Sect. 2 I will present a basic introduction to the Zeeman effect on the Stokes profiles of spectral lines. In Sect. 3 I will outline the current techniques for the modelling of magnetic fields; in Sect. 4 I will review the recent statistical results on the magnetic geometries of CP stars, and 

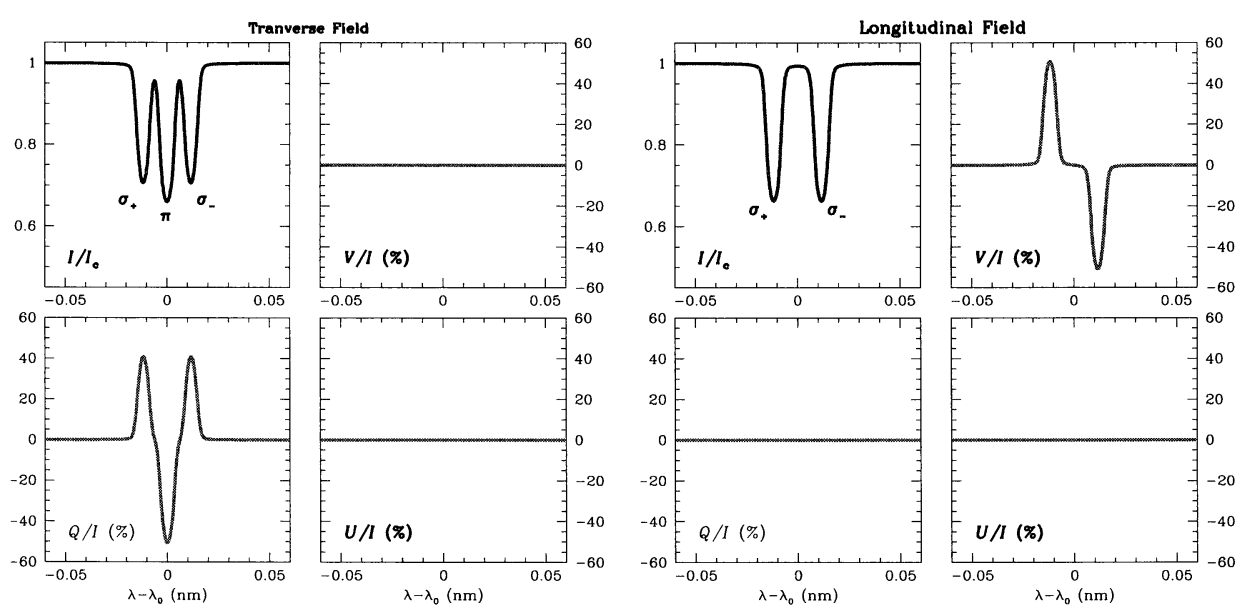

Figure 1. Stokes profiles of an optical spectral line characterised by a normal Zeeman pattern, formed in an element of a stellar surface characterised by a $10 \mathrm{kG}$ magnetic field perpendicular to the line of sight (left panels) and parallel to the line of sight (right panels)

in Sect. $5 \mathrm{I}$ will outline a new research project aimed at finding relationships between stellar magnetic fields and a star's evolutionary state.

\section{Polarization: what does it tell us?}

Polarization is described through the Stokes parameters $I$ (the 'usual' intensity), $Q$ and $U$ (which describe the linear polarization), and Stokes $V$ (which describes the circular polarization). The phenomena of polarization are connected with the possibility that is left to the electric field vector of spanning the plane perpendicular to the direction of propagation. Polarization originates whenever any kind of anisotropy occurs in the radiative source, e.g., scattering by matter, presence of collimated beams of particles, or presence of a magnetic field, thus in many different physical processes of emission of photons, and in many different kinds of astrophysical objects. Polarimetric observations provide information not only on how intensity depends on position, wavelength, and time, but also on how photons oscillate.

The observation and analysis of the properties of polarized radiation is the most direct diagnostic tool for the study of stellar magnetic fields. To start with, let us just remind that a spectral line characterised by a normal Zeeman pattern (triplet) formed in presence of a magnetic field directed perpendicularly to the line of sight will appear split into three components. A $\pi$ component is formed at the line rest wavelength, and is linearly polarized in the direction parallel to the magnetic field. Two $\sigma$ components appear polarized in the direction perpendicular to the line of sight at wavelengths corresponding to the rest wavelength increased and decreased, respectively, by an amount equal to the Larmor wavelength. In the presence of a magnetic field oriented along the 

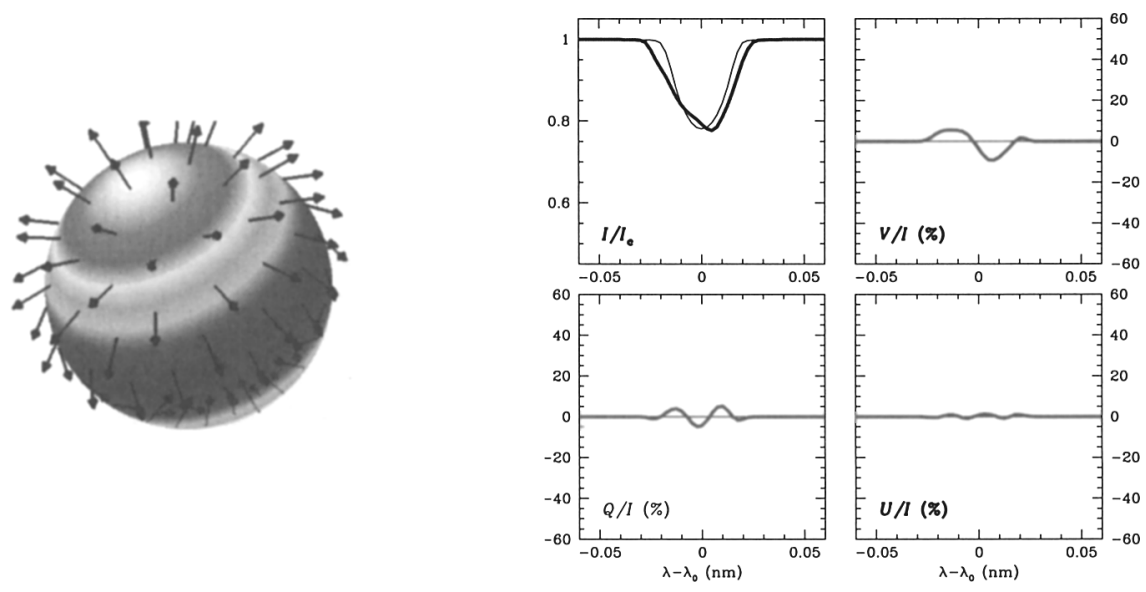

Figure 2. Thick solid lines show Stokes profiles of a spectral line characterised by a normal Zeeman pattern integrated over the stellar disk for a $10 \mathrm{kG}$ dipolar magnetic field as shown to the left side of the panels. The star is rotating with $v \sin i=10 \mathrm{kms}^{-1}$ about a rotation axis perpendicular to the line of sight. The thin solid lines refer to the non magnetic case.

line of sight, the spectral line appears split into two $\sigma$ components circularly polarized in opposite directions. These two situations are illustrated in Fig. 1. In the more general case of a magnetic field arbitrarily oriented with respect to the line of sight, the spectral line will appear split into three components elliptically polarized. In general, one can say that circular polarization measurements give information on the longitudinal component of the magnetic field (i.e. the component of the field along the line of sight). Linear polarization is instead sensitive to the transverse components of the magnetic field (i.e. the components perpendicular to the line of sight). Information on the magnetic strength is best obtained through the measurement of the Zeeman splitting on Stokes $I$.

In the stellar case, one has to consider a magnetic field that changes across the visible stellar disk, and to add up polarization signals coming from stellar disk elements characterised by different magnetic field orientations and strengths. The sum of all these polarization signals will tend to cancel out, thus in the 'stellar case' one has to expect a polarization signal considerably damped with respect to the 'local' case. In general, the more complex the field, the smaller the integrated polarization signal. In the stellar case, one has to consider also the effect of rotational Doppler broadening. The Zeeman splitting in Stokes $I$ tends to be washed out by the rotational Doppler broadening, and measurements of magnetically split lines are possible only in those few stars with strong magnetic field and very low $v \sin i$ values. Figure 2 shows an example of the Stokes profiles predicted for a star characterised by a simple dipolar morphology. It is interesting to note that the rotational Doppler effect washes out the Zeeman splitting, but does not necessarily damp the disk-integrated polarization. Due to the star's rotation, the polarization signals coming from different stellar regions 
are differentially wavelength shifted, so that for certain magnetic configurations it is less likely that they cancel out in a rotating star than in the case of zero $v \sin i$.

\section{Modelling techniques}

The problem of modelling of stellar magnetic fields consists of interpreting a time series of Stokes profiles like those shown in the right panel of Fig. 2 in terms of magnetic maps like the one shown in the left panel of the same Figure. This inversion problem is in principle best approached via Zeeman Doppler Imaging techniques (ZDI; Semel, 1989; Donati et al. 1989; Piskunov \& Kochukhov 2002). ZDI is aimed at reconstructing from a time series of Stokes profiles the stellar magnetic and element distribution maps with no a priori assumptions concerning their geometry. However, ZDI techniques can only be applied to spectropolarimetric data characterised by high resolution and extremely high $\mathrm{S} / \mathrm{N}$ ratio. Figure 2 refers to a particularly favourable magnetic configuration that produces a relatively high polarization signal. Real observations of Stokes profiles in magnetic stars exhibit polarization signals that in some cases are just barely distinguishable from photon noise, especially as far as Stokes $Q$ and $U$ are concerned (see Wade et al. 2000, which presents the richest collection of Stokes profiles obtained so far for CP stars). Only a few of the presently available data are suitable for the application of ZDI techniques (see Kochukhov \& Piskunov 2002 for the data requirements in terms of spectral resolution and S/N ratio). So far, the results of ZDI are probably of greater interest in order to define relationships between stellar magnetic fields and chemical element distribution in individual stars (e.g., Kochukhov et al. 2002b), but are not numerous enough as to define statistical properties of the magnetic morphologies (e.g., relationships between magnetic geometries and strengths and other stellar parameters.)

As a matter of fact, most of the data available in the literature are given in terms of estimates of magnetic observables, i.e., some quantities related to the geometry of the magnetic field, obtained from the the low order moments of the Stokes profiles about the line centre. An example is the mean longitudinal field, i.e., the average of the component of the magnetic field along the line of sight, which is obtained from the first order moment of Stokes $V$ about the line centre. Another popular magnetic observable is the mean magnetic field modulus, which is obtained from the measurement of the Zeeman splitting in Stokes I. The major advantage of these observing techniques is that basic information on the magnetic geometry can be obtained from relatively low resolution and low $\mathrm{S} / \mathrm{N}$ ratio spectra. Mathys $(1991,1995 \mathrm{a}, 1995 \mathrm{~b})$ has been active in developping the so called moment technique and gathering a large collection of data. Thus an attractive alternative modelling technique for stellar magnetic field is based on the combined interpretation of all these magnetic observables (see, e.g., Bagnulo et al. 2000 and references therein).

The statistical properties of CP stars presented in next Section have been found from the analysis of these magnetic observables. 


\section{Modelling results}

One of the most interesting results obtained in the last few years is the finding of a link between magnetic morphology and stellar rotation periods by Landstreet \& Mathys (2000). Assuming that the magnetic morphology is axisymmetric, they have shown that slow rotating stars (i.e., stars with a rotation period longer than about 1 month) have the magnetic axis tilted at a small angle with respect to the rotation axis.

Finding a relationship between stellar rotation and magnetic strength and geometry may suggest that a dynamo effect is sustaining to some extent the magnetic field. However, Stẹpień \& Landstreet (2002) presented an interpretation in terms of a fossil field, that can be outlined as follows. First, recall the theory of Stẹpien (2000) that the longest period magnetic stars lose their angular momentum during the pre-main sequence phase through magnetised wind after the dissipation of the circumstellar disk. Stępien \& Landstreet (2002) have further argued that magnetic structures with axis tilted at a small angle with respect to the rotation axis are the most efficient ones to dissipate the circumstellar disk. Thus, they conclude that stars with magnetic axis nearly parallel to the rotation axis have undergone a longer period during which the magnetised wind is effective in slowing down the star, which may eventually reach the Zero Age Main Sequence with a rotation period of months or longer.

Finding further statistical properties of magnetic CP stars requires a more general framework not limited to axisymmetric configurations. Here I present some statistical results obtained within the framework of a dipole + quadrupole field arbitrarily oriented presented in Landolfi et al. (1998), and based on the analysis of a sample of strongly magnetic CP stars nearly coincident with that studied by Landstreet \& Mathys (2000). It should be noted that this sample is not necessarily representative of all magnetic CP stars, being biased toward relatively slowly rotating and strong field stars.

Let us consider the angle $\beta_{\mathrm{Q}}$ between the rotation axis and the direction perpendicular to the plane identified by the unit vectors that define the quadrupolar component, as shown in the left panel of Fig. 3. Bagnulo et al. (2002a) have found striking evidence for this angle being near to $90^{\circ}$ in stars with rotation period of a few days or shorter. This situation is illustrated in the remaining panels of Fig. 3, that shows the histogram of the $\beta_{\mathrm{Q}}$ for slow rotating stars (middle panel) and for fast rotating stars (right panel), compared to the histograms expected from random distributions of the magnetic morphologies. A more specific feature has been also suggested by Bagnulo et al. (2002a). It concerns the angle $d$ between the direction perpendicular to the plane identified by the dipole and rotation axis, and the direction perpendicular to the quadrupolar plane, which tends to be small for fast rotating stars and large for slower rotating stars. In the left panel of Fig. 4, $R D$ indicates the plane identified by the rotation axis and the dipole axis, and the direction perpendicular to this plane is indicated by $\mathrm{A}$. For many fast rotating stars, the point $\mathrm{Q}$, that indicates the direction perpendicular to the quadrupole plane, is concentrated in a small cap around A ( $\sin d \simeq 0$ ). For slow rotating stars, the point $\mathrm{Q}$ is concentrated in a small ring near to the plane $R D(\sin d \simeq 1)$. There is an indication that $d$ increases with the star's rotational velocity (right panel of Fig. 4). There is no obvious physical interpretation of this finding, which has to be in fact be confirmed by means of 

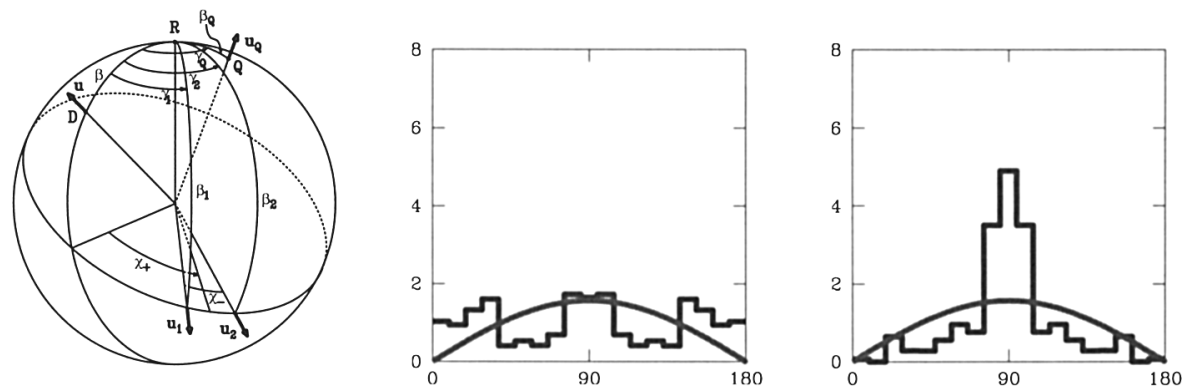

Figure 3. Left panel: the definition of the angle $\beta_{\mathrm{Q}}$ (from Bagnulo et al., 2002a); middle panel: histogram of $\beta_{Q}$ for stars with rotation period $<10 \mathrm{~d}$; right panel: histogram of $\beta_{Q}$ for stars with rotation period $>10 \mathrm{~d}$. The sinusoidal curves represent the histograms expected from a random distribution of the magnetic configurations
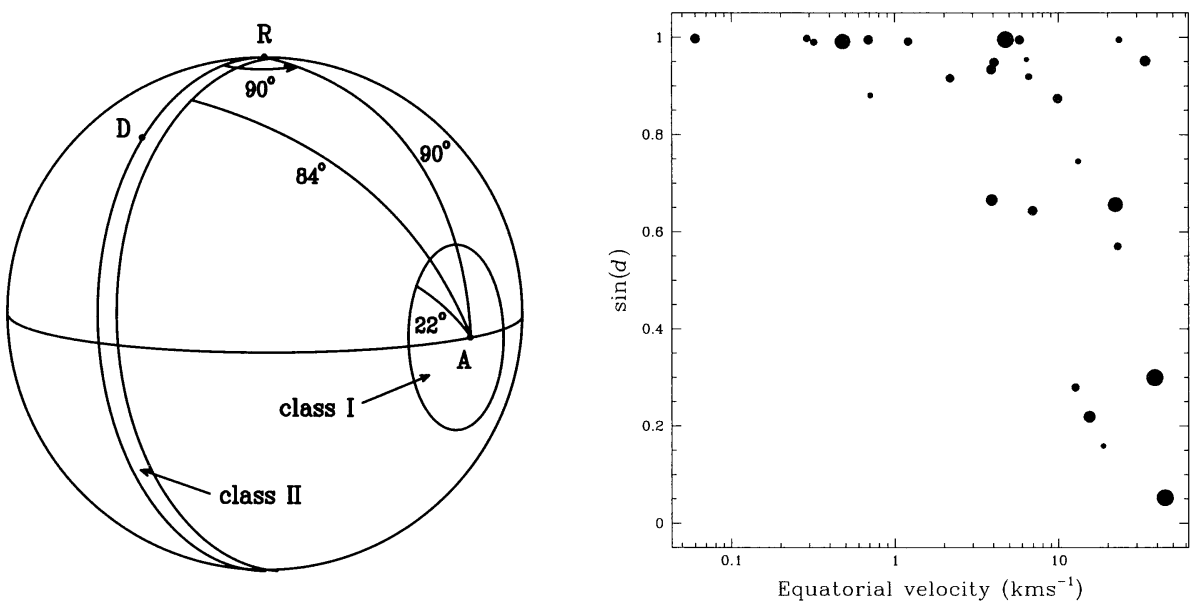

Figure 4. Left panel: for many fast rotating stars (class I stars as defined in Bagnulo et al. 2002a), the point $\mathrm{Q}$, that indicates the direction perpendicular to the quadrupole plane, is concentrated in a small cap around $\mathrm{A}$, which indicates the direction perpendicular to the plane identified by the quadrupole. For slow rotating stars (class II stars), the point $\mathrm{Q}$ is concentrated in a small ring near to the plane $R D$. Right panel: the complete scatter plot of $\sin d$ versus the star's rotational velocity. Dot size is proportional to the reliability of the model (i.e., proportional to $1 / \chi^{2}$ ). 

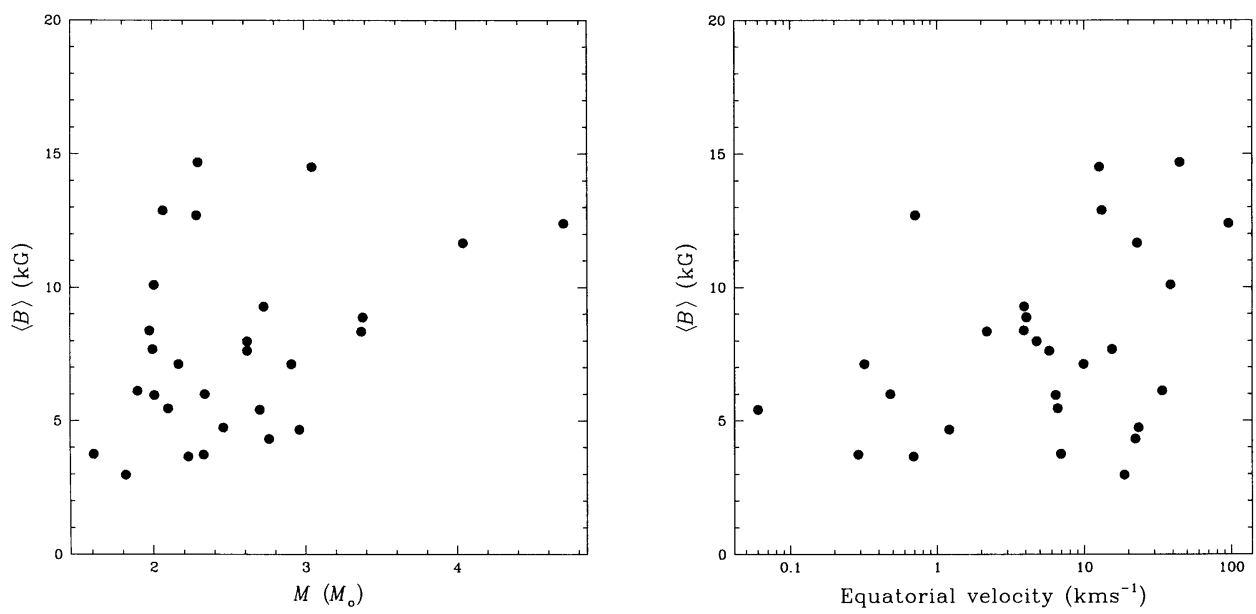

Figure 5. Right panel: mean field modulus (averaged over the rotation cycle) vs. the stellar equatorial velocity. Left panel: mean field modulus (averaged over the rotation cycle) vs. the stellar mass. In both plots, Babcock's star (with a mean field modulus $>30 \mathrm{kG}$ ) is not included.

more sophisticated modelling techniques based on the inversion of Stokes profiles rather than the magnetic observables. It should be reminded that the modelling of the magnetic observables is still quite an indirect technique, and that firm results can only come after a direct comparison of observed vs. model profiles.

Finally, we recall that Mathys et al. (1997) and Hubrig et al. (2000) have noted a (loose) relationship between mean field modulus and rotation period (see also Bagnulo et al. 2002a), and between mean field modulus and stellar temperature. In Fig. 5 I show the plots of mean field modulus averaged over the rotation cycle vs. the equatorial velocity, and of the mean field modulus averaged over the rotation cycle vs. the stellar mass. Fig. 5 includes stars for which observations of mean field modulus are not available: the relevant estimates are obtained from the model of other magnetic observables.

\section{Searching for links between magnetic field and stellar evolution}

The problem of the origin of the magnetic field is an open one. It is common to generically assume a fossil origin, either the relic of the field pervading the interstellar medium from which the star formed, or that remaining from a dynamo acting in the convective Hayashi phase of pre-main sequence evolution. According to the (possibly less popular) core dynamo theory, instead, the magnetic field is generated in the star's rotating convective core and maintained by a contemporary dynamo action (see, e.g., Moss 2001).

Both theories face serious difficulties. The fossil theory requires that fields are stable on timescales of more than a few $\times 10^{8}$ years; unfortunately the theory 
of stability is still unsatisfactory (Moss 2001). From the core-dynamo theory one expects a strong relationship between magnetic strength and rotation period, which in fact is not observed. A major issue common to both theories is why some stars are magnetic and why other ones are not. Being formed from the same interstellar cloud, fossil theory predicts that all A and B stars of a given open cluster should be either all magnetic or all non magnetic. However, the observational evidence is that within individual open clusters, some $\mathrm{A}$ and $\mathrm{B}$ stars are magnetic, but most of them are not, just like in the field. From the dynamo theory, one should simply expect that all A and B stars, either in clusters or in the field, be magnetic.

Recently, Hubrig et al. (2000, ApJ 539, 352) have studied a sample of strongly magnetic CP stars (in fact, similar to the samples studied by Landstreet \& Mathys 2002, and by Bagnulo et al. 2002a), and concluded that, for stellar masses $\leq 3 M_{\odot}$, magnetic stars are concentrated toward the centre of the main sequence band. Hence, they suggest that the magnetic field forms after the star has spent at least $1 / 3$ of its life on the main-sequence. Clearly, Hubrig et al.'s interpretation, if confirmed, would add up an additional problem to the theories of the origin of the magnetic field, and would also leave one with no satisfactory explanation for the slow rotation of magnetic CP stars, particularly for the very slow rotators that represent most of the sample they used. However, it should be noted that an opposite result has been found by Gomez et al. (1998), who have constructed the H-R diagram of about $1000 \mathrm{CP}$ stars, and concluded that most of them lie on the Main Sequence occupying the whole width of it, like normal stars in the same range of spectral types. Under the assumption that most chemically peculiar stars are magnetic, Gomez et al.'s result suggests that magnetic stars are also present throughout the Main Sequence.

In an attempt to clarify the relationship between the occurrence of magnetic phenomena and the stellar evolution, J.D. Landstreet, T. Szeifert, G. Wade, and I have started a campaign of spectropolarimetry of A and B stars in open clusters. This research is based on the observations of $\mathrm{H}$ Balmer lines at low resolution and in circular polarization, by means of the FORS1 instrument at the ESO VLT (see Bagnulo et al. 2002b for the description of the observing technique). The number of observations obtained so far are still not statistically meaningful, but we hope that after a few further observing runs, we will be able to set a firm constraint to the evolutionary state of the magnetic CP stars.

For the time being, it is of some interest to present the discovery of an exceedingly strong magnetic field in a member of the cluster NGC 2516, the chemically peculiar star HD 66318. In February 2002 (our first observing run with FORS1), the mean longitudinal field measured on the $\mathrm{H} \alpha$ profiles (see Fig. 6) was about $+4500 \mathrm{kG}$. In a high-resolution spectroscopic follow up obtained in March with $C E S$ at the $3.6 \mathrm{~m}$ telescope of La Silla, and in May 2002 with $U V E S$ at the $V L T$ unit Kueyen, we have measured a nearly constant mean field modulus of about $15 \mathrm{kG}$ (i.e., the mean field modulus did not change in a two months interval of time). We also measured a rotational velocity consistent with zero. This suggests that HD 66318 is either seen pole-on, or is a very long-period magnetic star. 

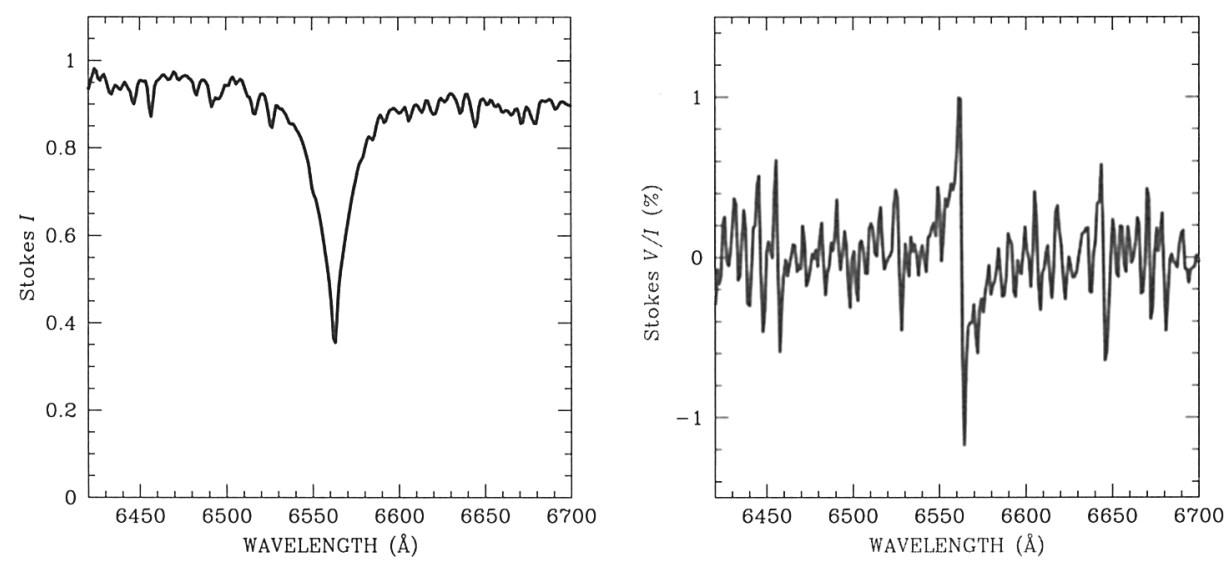

Figure 6. $\mathrm{H} \alpha$ Stokes $I$ (left panel) and Stokes $V$ (right panel) profiles obtained with FORS1 at the VLT at low resolution $(R \simeq 2400)$, for HD 66318, member of the open cluster NGC 2516. The star was previously known to be chemical peculiar of type, but no magnetic field had been detected prior to these observations.

\section{Conclusions}

Some $5-10 \%$ of early-type stars have a strong, fairly homogeneous magnetic field, the origin of which is currently lacking a satisfactory explanation. The presence of the magnetic field has deep consequences for the star as a whole, causing loss of angular momentum in the pre-main sequence phase, and on its atmospheric structure, leading to an inhomogeneous distribution of the chemical elements. These features cause magnetic CP stars to be one of the most important laboratories for testing our understanding of stellar astrophysics.

\section{Discussion}

LINSKY: Non thermal gyrosynchrotron emission is detected in many magnetic $\mathrm{CP}$ stars in young clusters like Orion and Scorpius-Centaurus, but few in the field. This supports but does not prove that the chemical peculiar stars are young and that the magnetic field is like a fossil field. Do you agree?

BAGNULO: Yes, this finding suggests that some magnetic CP stars are young, although it may not always be easy to associate the sources to their optical counterparts.

WEISS: A fairly detailed survey of open clusters by Maitzen et al. (in Vienna) with different ages using photometric techniques did not indicate a significant dependency of the occurrence of CP2 stars with cluster age. A similar conclusion was drawn by $\mathrm{S}$. Hubrig in her $\mathrm{PhD}$ thesis.

BAGNULO: To extend Maitzen et al.'s result to magnetic stars one has to assume that all CP2 stars are magnetic; a reasonable hypothesis indeed (see 
Shorlin et al. 2002).

STEINER: You showed strongly asymmetric observed and synthetic Stokes $V$ profiles. In solar surface observations this typically indicates velocity and possibly magnetic field gradients. Are these asymmetries here simply a result of stellar rotation? The addition of an outflow of plasma would add to Stokesprofiles asymmetries and would correspondingly complicate the interpretation.

BAGNULO: Stokes $V$ profile (see Fig. 2) is not symmetric about the line centre because of the stellar rotation. We do not hypothesise the presence of plasma outflows in CP stars, and the local contribution to Stokes $V$ is symmetric about the line centre. Since the magnetic morphology is not symmetric about the rotation axis, the contributions that are blue-shifted do not have in general an identical red-shifted counterpart, thus the integrated profile is not symmetric about the line centre.

COHEN: What is the relationship between Ap stars and magnetic white dwarfs? BAGNULO: A reasonable working hypothesis is that Ap stars are the progenitors of at least some magnetic white dwarfs.

KHALACK: What is a mean value of the magnetic field modulus for the sample of analysed stars? Has the relation longitudinal field over field modulus the same value for the different samples of stars?

BAGNULO: The stars of the samples that have been the object of the statistical studies discussed here have a mean field modulus between 3 and $15 \mathrm{kG}$ (with the exception of Babcock's star that has a mean field modulus $>30 \mathrm{kG}$ ). The mean longitudinal field is a quantity that strongly depends on the orientation of the rotation axis with respect to the line of sight, and on the rotation phase. It could be zero even for very strong magnetic stars, thus I did not do a statistical study of the ratio between longitudinal field and mean field modulus.

\section{References}

Alecian, G., \& Stift, M.J., 2002, A\&A,387, 271

Babcock, H.W., 1949, the Observatory, 69, 191

Babel, J., \& Lanz, T., 1992, A\&A, 263, 232

Bagnulo, S., Landolfi, M., Mathys, G., \& Landi Degl'Innocenti, M. 2000, A\&A, 343, 865 A\&A, 358, 929

Bagnulo, S., Wade, G.A., Donati, J.-F., Landstreet, J.D., Leone, F., Monin, D.N., \& Stift, M.J., 2001, A\&A, 369, 889

Bagnulo, S., Landi Degl'Innocenti, M., Landolfi, M., \& Mathys, G., 2002a, A\&A, (in press)

Bagnulo, S., Szeifert, T., Landstreet, J.D., Wade, G.A., \& Mathys, G., 2002b, A\&A, 389, 191

Cowley, C. R., Hubrig, S., Ryabchikova, T. A., Mathys, G., Piskunov, N., \& Mittermayer, P., 2001, A\&A, 367, 939

Donati, J.-F., Semel, M., \& Praderie, F., 1989, A\&A, 225, 467 
Gomez, A.E., Luri, X., Grenier, S., Figueras, F., North, P., Royer, F., Torra, J., \& Mennessier, M.O., 1998, A\&A, 336, 953

Hubrig, S., \& Castelli, F., 2001, A\&A, 375, 963

Hubrig, S., North, P., \& Mathys, G., 2000, ApJ, 539, 352

Kochukhov, O., Bagnulo, S., \& Barklem, P.S., 2002a, ApJ, 578, 75

Kochukhov, O., Piskunov, N., Ilyin, I., Ilyina, S., \& Tuominen, I., 2002b, A\&A, 389,420

Landolfi, M., Bagnulo, S., \& Landi Degl'Innocenti, M., 1998, A\&A, 338, 111

Landstreet, J.D., \& Mathys, G. 2000, A\&A, 359, 213

Leroy, J.L., Bagnulo, S., Landolfi, M., \& Landi Degl'Innocenti, E., 1994, A\&A, 284,174

Mathys, G. 1994, A\&AS, 108, 547

Mathys, G. 1995a, A\&A, 293, 733

Mathys, G. 1995b, A\&A, 293, 746

Mathys G., Hubrig S., Landstreet J.D., Lanz T., \& Manfroid J., 1997, A\&AS 123,353

Michaud, G., 1970, ApJ, 160, 641

Moss, D., 2001, in: Magnetic fields across the Hertzsprung-Russell Diagram, Mathys, G., Solanki, S.K., \& Wickramasinghe, D.T., A.S.P. Conference Series vol. 248, p. 305

North, P., 1998, A\&A, 334, 181

Piskunov, N., \& Kochuckov, O., 2002, A\&A, 381, 736

Preston, G.W., 1974, ARA\&A, 12, 257

Ryabchikova, T., LeBlanc, F., \& Wade, G.A., 2002, this volume

Semel, 1989, A\&A, 225, 456

Shorlin, S.L.S., Wade, G.A., Donati, J.-F., Landstreet, J.D., Petit, P., Sigut, T.A.A., \& Strasser, S., 2002, A\&A, 392, 637

Stępien, K., 2000, A\&A, 353, 227

Stȩpien, K., \& Landstreet, J.D., 2002, A\&A, 384, 554

Stibbs, D.W.N., 1950, MNRAS, 110, 395

Wade, G., Donati, J.-F., Landstreet, J.D., \& Shorlin, S.L.S., 2000, MNRAS, 313,851 